\title{
High transverse momentum physics in heavy ion collisions
}

\author{
Néstor Armesto*i \\ Departamento de Física de Partículas and IGFAE, Universidade de Santiago de Compostela, \\ Spain \\ E-mail: nestor@fpaxp1.usc.es
}

Our current understanding on high transverse momentum phenomena in heavy ion collisions is reviewed. Both single inclusive particle production and more differential observables are considered. The properties of the probed medium extracted from measurements at RHIC, and the perspectives for the LHC, are discussed.

International Europhysics Conference on High Energy Physics

July 21st - 27th 2005

Lisboa, Portugal

\footnotetext{
${ }^{*}$ Speaker.

†Investigador Ramón y Cajal of Ministerio de Educación y Ciencia of Spain; financial support of CICYT of Spain under project FPA2002-01161 is acknowledged. I thank the organizers for their invitation to give this talk in such a nice conference. I also thank M. Cacciari, L. Cunqueiro, A. Dainese, P. Jacobs, F. Krauss, C. Loizides, D. Magestro, A. Morsch, J. Schukraft and U. A. Wiedemann for many useful discussions on all these subjects, and C. A. Salgado for discussions and a critical reading of the manuscript.
} 


\section{Introduction}

Much attention has been lately devoted to high transverse momentum physics in heavy ion collisions (HICs). A high momentum parton produced in a HIC is a hard probe $[1,2]$ of the medium produced in such collision. A quark or a gluon traversing the unknown medium will lose energy due to elastic scattering and/or induced gluon radiation. Both massless and massive probes (heavy quarks) are available, together with a control of the benchmark provided by real and virtual photons which do not interact with the medium. The requirement of a high transverse momentum $p_{T} \gg \Lambda_{\mathrm{QCD}}$ provides a hard scale. This makes it possible to compute the benchmark (pp and $\mathrm{p}-\mathrm{A}$ collisions) using perturbative techniques.

I will refer to a region of transverse momentum large enough to ensure that what traverses the medium is a parton which fragments outside the medium i.e. in the vacuum. This is not the case in the intermediate $p_{T}(<4 \div 7 \mathrm{GeV})$ region at RHIC $[3,4,5,6]$, as indicated e.g. by the baryon-tomeson anomaly. At the LHC even larger transverse momenta should be affected by fragmentation inside medium. I will discuss only the main conclusions extracted from these studies, the relevant references can be found in the reviews except for the most recent developments.

\section{Medium-induced gluon radiation}

Medium-induced gluon radiation (Fig. 1; for reviews, see [7, 8, 9]) is usually assumed to be the dominant process underlying jet quenching at high parton energies (which correspond to high $p_{T}$ at mid-rapidities where most of the experimental data exist). It implies the energy degradation of the leading particle (the quenching itself), a broadening of the associated parton shower (sometimes called jet heating) and an increase of the associated hadron multiplicity. The dominant contribution comes from the rescattering of the produced gluon with the medium. The medium is characterized by the product density of scattering centers times cross section of the parton with the scattering center. Under certain limits, it leads to the prediction that the energy loss is independent of the energy of the radiating parton, and proportional to the mentioned product, to the color charge of the parton and to the square of the length of the medium.

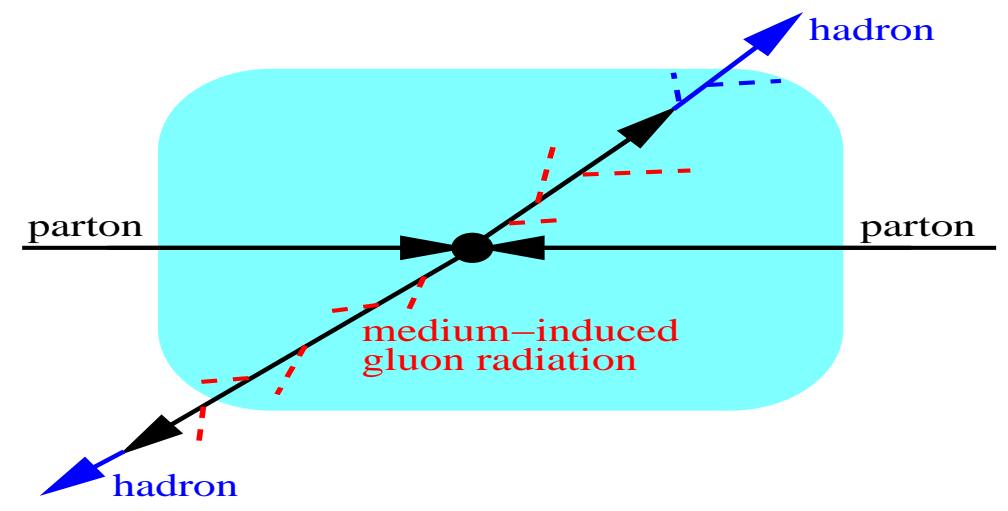

Figure 1: Jet quenching by radiation off fast partons produced inside a medium.

Until recently, the main focus of jet quenching studies has been on the suppression of single particle spectra in nucleus-nucleus collisions. Models which implement the available formalisms 
taking into account in a realistic way the collision geometry, are successful in describing such suppression and its centrality and energy dependence, as well as the observed disappearance of back-to-back correlations $[3,4,5,6]$. In this way, a lower bound on the density of the medium has been obtained. Nowadays the main interest lies in the characteristic features of color charge and mass dependence of the quenching (see [10] and references therein), and of the correlations in associated radiation and its coupling with the medium [11], see [12, 13, 14, 15] and refs. therein.

\section{Situation at RHIC}

The description of the suppression of single particle spectra and the disappearance of backto-back correlations at RHIC within the radiative energy loss framework demands [12, 13, 14, 15] a very large density of the medium, which can be characterized either by the average squared momenta transferred from the medium to the parton per mean free path, the so-called transport coefficient $\hat{q}=4 \div 14 \mathrm{GeV}^{2} / \mathrm{fm}$, or alternatively by a large initial gluon density $d N_{g} / d y=1000 \div$ 3500 [16]. The centrality dependence is successfully reproduced with a medium with a realistic geometry. The effects of the dilution of the medium due to expansion are taken into account consistently, through a redefinition of the transport coefficient/initial gluon density.

The opacity of the medium at RHIC is such that high- $p_{T}$ particle production becomes surface dominated, with the corresponding difficulty to establish an upper bound for $\hat{q}$ or $d N_{g} / d y$. The observed re-appearance of the backward jet for high- $p_{T}$ triggers [17] may provide further constraints $[18,19]$. Another opportunity comes from the study of heavy flavor production. The mass of heavy quarks reduces the medium-induced radiation [10], offering a new test of the picture underlying jet quenching and new opportunities to characterize the medium. Unfortunately, heavy hadrons are not identified in the heavy ion environment at RHIC, and available information comes from non-photonic electrons coming dominantly from D/B decays. New data [20,21] extend up to $p_{T}^{e} \sim 10 \mathrm{GeV}$ and show a strong suppression similar to that of light hadrons, but uncertainties in the relative charm/beauty contribution [16, 22] obscure possible conclusions.

A final topic is the possible modifications of radiation coming from the flow in the medium [11, 14]. While data at small $p_{T}$ have been subject to a strong debate $[13,14,15]$, studies at larger transverse momenta may offer new possibilities to characterize the medium beyond a simple density. They may also help to enhance the elliptic flow at large $p_{T}$ which in jet quenching formalisms is predicted to be smaller than found in data [14].

\section{Perspectives for the $\mathrm{LHC}$}

At the LHC, hard transverse momentum particles and jets will be produced abundantly [2, 23]. The study of large samples of e.g. jets with $E_{T}=100 \mathrm{GeV}$ will open new possibilities. There are several proposals to use the characterization of jet profiles to get information about the density and dynamical properties of the medium [11,14], and also to study heavy flavor production to test the color charge and mass dependence of radiative energy loss [10].

The main difficulties $[2,23]$ in the use of jets in HIC at the LHC come from the determination of the energy of the jet. The true energy of the jet (of the parent parton) is required to get the fraction of energy loss, and to characterize the jet profile and fragmentation functions to be compared with 
those in pp collisions. The high multiplicity background in HIC demands the use of small size cones, $R=\sqrt{\eta^{2}+\phi^{2}}=0.3 \div 0.4$ for the jet to stand clearly over the background. Typically $\sim 80$ $\%$ of the energy of the jet lies within such cone sizes in $\mathrm{p}^{-} \mathrm{p}$ collisions (where $R=0.7 \div 1$ are the usual values for the jet definition). But there is no theoretical tool yet to determine how the energy lost in the medium is redistributed. Were it thrown to large $R$, the jet energy determination would become impossible. Also the small cone size enhances event-by-event fluctuations coming from e.g. the ratio charged/neutral or the energy deposition near the border of the defined cone.

A possible way out is to use jets balanced by a particle with no final state interaction and easy to identify, like a $\gamma, \mathrm{a} \mathrm{Z}^{0}$ or a $\mathrm{W}^{ \pm}$. The caveat comes from the small yield of such events $[2,23]$, but even a small sample would be useful to calibrate the jet-finding algorithms and the models used to simulate medium effects. Experimental studies and further theoretical work will be the main effort in the near future in order to overcome these difficulties.

\section{References}

[1] A. Accardi et al., Chapter 1: (pdf's, shadowing and pA collisions) of the CERN Yellow Report on Hard probes in Heavy-Ion Collisions at the LHC, CERN-2004-009 [arXiv:hep-ph/0308248].

[2] A. Accardi et al., Chapter 2: (Jet Physics) of the CERN Yellow Report on Hard probes in Heavy-Ion Collisions at the LHC, CERN-2004-009 [arXiv:hep-ph/0310274].

[3] K. Adcox et al. [PHENIX Collaboration], Nucl. Phys. A 757 (2005) 184.

[4] I. Arsene et al. [BRAHMS Collaboration], Nucl. Phys. A 757 (2005) 1.

[5] B. B. Back et al. [PHOBOS Collaboration], Nucl. Phys. A 757 (2005) 28.

[6] J. Adams et al. [STAR Collaboration], Nucl. Phys. A 757 (2005) 102.

[7] R. Baier, D. Schiff and B. G. Zakharov, Ann. Rev. Nucl. Part. Sci. 50 (2000) 37.

[8] M. Gyulassy, I. Vitev, X. N. Wang and B. W. Zhang, arXiv:nucl-th/0302077.

[9] A. Kovner and U. A. Wiedemann, arXiv:hep-ph/0304151.

[10] N. Armesto, A. Dainese, C. A. Salgado and U. A. Wiedemann, Phys. Rev. D 71 (2005) 054027.

[11] N. Armesto, C. A. Salgado and U. A. Wiedemann, Phys. Rev. Lett. 93 (2004) 242301.

[12] U. A. Wiedemann, Eur. Phys. J. C 43 (2005) 215.

[13] I. Vitev, arXiv:hep-ph/0503221.

[14] C. A. Salgado, arXiv:hep-ph/0510062.

[15] X. N. Wang, arXiv:nucl-th/0511001.

[16] M. Djordjevic, M. Gyulassy, R. Vogt and S. Wicks, arXiv:nucl-th/0507019.

[17] D. Magestro [STAR Collaboration], arXiv:nucl-ex/0510002.

[18] A. Dainese, C. Loizides and G. Paic, arXiv:hep-ph/0511045.

[19] I. Vitev, arXiv:hep-ph/0511237.

[20] J. Bielcik [STAR Collaboration], arXiv:nucl-ex/0511005.

[21] S. S. Adler et al. [PHENIX Collaboration], arXiv:nucl-ex/0510047.

[22] N. Armesto, M. Cacciari, A. Dainese, C. A. Salgado and U. A. Wiedemann, arXiv:hep-ph/0511257.

[23] P. M. Jacobs and M. van Leeuwen, arXiv:nucl-ex/0511013. 